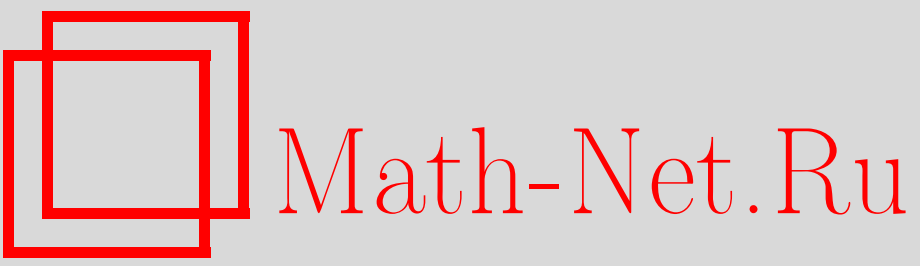

В. В. Дубровский, В. В. Дубровский (мл.), К теореме существования решения в обратной задаче спектрального анализа, УМH, 2001, том 56, выпуск 1, 161-162

DOI: https://doi.org/10.4213/rm364

Использование Общероссийского математического портала Math-Net.Ru подразумевает, что вы прочитали и согласны с пользовательским соглашением

http://www. mathnet.ru/rus/agreement

Параметры загрузки:

IP : 3.95 .254 .165

26 апреля 2023 г., 18:04:11 


\title{
К ТЕОРЕМЕ СУЩЕСТВОВАНИЯ РЕШЕНИЯ В ОБРАТНОЙ ЗАДАЧЕ СПЕКТРАЛЬНОГО АНАЛИЗА
}

\author{
В.В.ДуБровский, В.В.ДуБРовский (мл.)
}

Пусть $\Pi=\{(x, y) \mid 0 \leqslant x \leqslant a, 0 \leqslant y \leqslant b\}$ и $\Pi_{4}=\left\{(x, y) \mid 0 \leqslant x \leqslant a \cdot 2^{-1}, 0 \leqslant y \leqslant b \cdot 2^{-1}\right\}$ - прямоугольники, где $a>0, b>0$. Рассмотрим в $l_{2}(\Pi)$ граничную задачу $M$ :

$$
-\Delta \varphi=\lambda \varphi,\left.\quad \varphi\right|_{\partial \Pi}=0 .
$$

Введем оператор $T_{\beta}=\int \lambda^{\beta} d E(\lambda)$, где $E(\lambda)$ - спектральное разложение единицы оператора $M, \beta>0$ и $\lambda^{\beta}>0$ при $\lambda>0$. Собственные числа $\lambda_{m, s}=\left(\pi^{2} a^{-2} m^{2}+\pi^{2} b^{-2} s^{2}\right)^{\beta}$, $m, s=\overline{1, \infty}$, оператора $T_{\beta}$ будем нумеровать в порядке возрастания их величин с помощью натурального индекса $n$. Соответствующие им ортонормированные функции оператора $T_{\beta}$ имеют вид $V_{m, s}=2^{-1}(a, b)^{-1} \sin \left(\pi b^{-1} m x\right) \sin \left(\pi b^{-1} s y\right)$. Обозначим $d_{n}$ расстояние от собственного числа $\lambda_{n}$ до остальной части спектра $\sigma\left(T_{\beta}\right)=\bigcup_{m, s=1}^{\infty} \lambda_{m, s}$ оператора $T_{\beta}$. Известно, что существует такая подпоследовательность натуральных чисел $\left\{n_{k}\right\}_{k=1}^{\infty}$, что $d_{n_{k}} \geqslant C_{1} n_{k}^{\beta-1}$, где $C_{1}>0$ и не зависит от индекса $n_{k}$.

Введем оператор умножения $P$ на вещественную, измеримую по Лебегу, существенно ограниченную по модулю функцию $p(x, y)((x, y) \in \Pi)$, которая удовлетворяет условиям:

$$
p(a-x, y)=p(x, y)=p(x, b-y)
$$

для почти всех $(x, y) \in \Pi$;

$$
\iint_{\Pi} p(x, y) \cos \left(2 \pi a^{-1} n x\right) d x d y=\iint_{\Pi} p(x, y) \cos \left(2 \pi b^{-1} n y\right) d x d y=0,
$$

$n=\overline{1, \infty}$. Пусть $\left\{\mu_{n}\right\}_{n=1}^{\infty}-$ собственные числа оператора $T_{\beta}+P$, занумерованные в порядке возрастания их величин с учетом кратности.

Лемма 1. Пусть $0<\delta<1,(1-\delta)\left(\beta-\frac{1}{2}\right)>1$, тогда на вертикальных прямых $\Gamma_{n_{k}}=\left\{\left(\lambda_{n_{k}}+\lambda_{n_{k+1}}\right) 2^{-1}+i \rho, \rho \in \mathbb{R}\right\}$ выполняется равенство:

$$
\begin{gathered}
\left\|(T-\lambda E)^{-1}\right\|_{1} \equiv \sum_{n=1}^{\infty}\left|\lambda-\lambda_{n}\right|^{-1}=\left(|\rho|+C_{4} n_{k}^{\beta-1}\right)^{-\delta} O\left(n_{k}^{\frac{1}{2}-(1-\delta)(\beta-1)}\right) \\
+\left(|\rho|+C_{4} n_{k}^{\beta-\frac{1}{2}}\right)^{-\delta} O\left(n_{k}^{1-(1-\delta)\left(\beta-\frac{1}{2}\right)}\right)
\end{gathered}
$$

где $C_{4}>0$ u $\|A\|_{1}$ означает ядерную норму оператора $A$.

Обозначим $f_{m_{l}}(\lambda)=\left[\prod_{j=1, j \neq l}\left(\lambda-\lambda_{m_{j}}\right) /\left(\lambda+\lambda_{m_{j}}\right)\right] \frac{1}{f_{m_{l}}}-$ ограниченные по модулю, аналитические функции в правой полуплоскости:

$$
f_{m_{l}}\left(\lambda_{m_{j}}\right)=\delta_{j_{l}}, \quad\left\|f_{m_{l}}(\lambda)\right\|_{\infty}=\sup _{\operatorname{Re} \lambda>0}\left|f_{m_{l}}(\lambda)\right|=\left|f_{m_{l}}^{-1}\right| .
$$

На подпоследовательность $\left\{m_{l}\right\}$ наложим ограничение:

$$
f_{m_{l}}^{-1}=O\left(n_{k}^{\beta-\frac{3}{2}-\alpha}\right)
$$

где $\alpha>0$ и $m_{l} \in\left\{n_{k-1}+1, n_{k-1}+2, \ldots, n_{k}\right\}$. 
Обозначим $\varphi_{m_{l}}(\lambda)=\int_{1}^{\lambda} f_{m_{l}}(\lambda) d \lambda$ и рассмотрим спектральное равенство при $m_{l} \in$ $\left\{n_{k-1}+1, n_{k-1}+2, \ldots, n_{k}\right\}$ :

$$
\begin{aligned}
\sum_{j=n_{k-1}+1}^{n_{k}} \varphi_{m_{l}}\left(\mu_{j}\right)= & \sum_{j=n_{k-1}+1}^{n_{k}} \varphi_{m_{l}}\left(\lambda_{j}\right)+\left(P V_{m_{l}}, V_{m_{l}}\right) \\
& -\frac{1}{6 \pi i} S_{p}\left\{\int_{\Gamma_{n_{k}}}-\int_{\Gamma_{n_{k-1}}}\right\} f_{m_{l}}(\lambda)\left(P(T-\lambda E)^{-1}\right)^{2} d \lambda+\cdots \\
\equiv & \sum_{j=n_{k-1}+1}^{n_{k}} \varphi_{m_{l}}\left(\lambda_{j}\right)+\left(P V_{m_{l}}, V_{m_{l}}\right)+\alpha m_{l}(p),
\end{aligned}
$$

которое записано в предположении

$$
\iint_{\Pi_{4}} p(x, y) \psi_{t}(x, y) d x d y=0
$$

при $t \neq m_{l}, \psi_{t}(x, y)=(a b)^{-1} \cos \left(2 \pi a^{-1} m x\right) \cos \left(2 \pi b^{-1} s y\right)$, где $\lambda_{t}=\left(\pi a^{-2} m^{2}+\pi^{2} b^{-2} s^{2}\right)^{\beta}$.

При $\beta>\frac{3}{2}$ и $\alpha>0$ можно построить такую подпоследовательность натуральных индексов $t_{q}$, что

$$
I=\sum_{q=1}^{\infty}\left|O\left(n_{t_{q}}^{-\alpha}\right)\right|=\sum_{q=1}^{\infty}\left|O\left(n_{t_{q}}^{\beta-\frac{3}{2}-\alpha}\right)\right| \cdot\left[\left|O\left(t_{q}^{\frac{3}{2}-\beta}\right)\right|+\left|O\left(n_{t_{q}}^{2-\beta-\frac{1}{2 \delta+2}}\right)\right|\right]<\infty .
$$

Обозначим

$$
\alpha_{0}(p)=\sum_{l=1}^{\infty}\left(\sum_{j=n_{t_{q-1}+1}}^{n_{t_{q}}}\left(\varphi_{m_{l}}\left(\mu_{j}\right)-\varphi_{m_{l}}\left(\lambda_{j}\right)\right) \psi_{m_{l}}\right),
$$

где $m_{l} \in\left\{n_{t_{q-1}}+1, n_{t_{q-1}}+2, \ldots, n_{t_{q}}\right\}, \alpha(p)=\sum_{l=1}^{\infty} \alpha_{m_{l}}(p) \psi_{m_{l}}$. Здесь $\alpha m_{l}(p)$ взяты в (4) при условии $k=t_{q}$.

Тогда

$$
\|\alpha(p)-\alpha(\tilde{p})\|_{\infty} \leqslant\|p-\tilde{p}\|_{\infty}\left(\|p\|_{\infty}+\|\tilde{p}\|_{\infty}\right) I=\gamma\|p-\tilde{p}\|_{\infty} .
$$

По принципу Стефана Банаха операторное уравнение

$$
\alpha_{0}-\alpha(p)=P
$$

где

$$
\alpha_{0}=\sum_{j=1}^{\infty}\left(\left(\xi_{m_{l}}-\sum_{j=n_{t_{q-1}+1}}^{n_{t_{q}}} \varphi_{m_{l}}\left(\lambda_{j}\right)\right)\right) \psi_{m_{l}}
$$

имеет единственное решение $p$ при $\alpha_{0} \in U(0, \varepsilon)$.

Теорема 2. Если $a^{2} b^{-2}$ - иррачиональное число, $\beta>\frac{3}{2} u\left\{\lambda_{m_{n}}\right\}-$ такая подпоследовательность собственных чисел оператора $T_{\beta}$, что выполнено (3), то существует $\varepsilon>0$, зависящее от $O\left(n_{k}^{-\alpha}\right)$, такое, ито в замкнутом шаре $U(0, \varepsilon)$ существует единственный потенциал $p$, удовлетворяющий условиям (1) и (2), и при условии $\alpha_{0} \in U(0, \varepsilon)$ выполняется равенство:

$\sum_{j=n_{t_{q-1}}+1}^{n_{t_{q}}}\left(\varphi_{m_{l}}\left(\mu_{j}\right)-\varphi_{m_{l}}\left(\lambda_{j}\right)\right)=\xi_{m_{l}}, \quad n=\overline{1, \infty}, \quad m_{l} \in\left\{n_{t_{q-1}}+1, n_{t_{q-1}}+2, \ldots, n_{t_{q}}\right\}$, $u$

$$
\iint_{\Pi_{4}} p(x, y) \psi_{t}(x, y) d x d y=0, \quad t \neq m_{l}, \quad l=\overline{1, \infty}
$$

